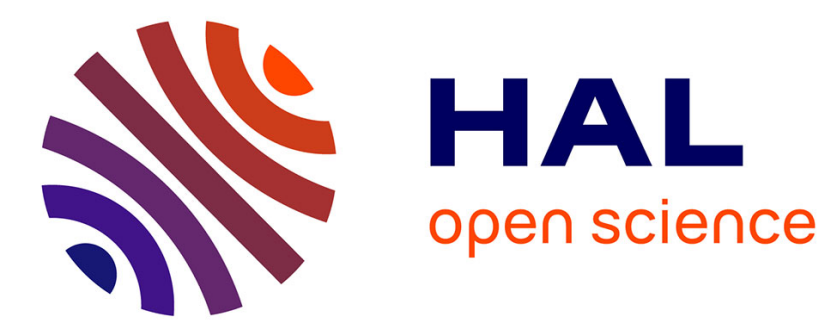

\title{
ON TOTALLY PERIODIC $\omega$-LIMIT SETS FOR MONOTONE MAPS ON REGULAR CURVES
}

Amira Mchaalia

\section{To cite this version:}

Amira Mchaalia. ON TOTALLY PERIODIC $\omega$-LIMIT SETS FOR MONOTONE MAPS ON REGULAR CURVES. 2021. hal-03018900v2

\section{HAL Id: hal-03018900 \\ https://hal.science/hal-03018900v2}

Preprint submitted on 10 Jun 2021

HAL is a multi-disciplinary open access archive for the deposit and dissemination of scientific research documents, whether they are published or not. The documents may come from teaching and research institutions in France or abroad, or from public or private research centers.
L'archive ouverte pluridisciplinaire HAL, est destinée au dépôt et à la diffusion de documents scientifiques de niveau recherche, publiés ou non, émanant des établissements d'enseignement et de recherche français ou étrangers, des laboratoires publics ou privés. 


\title{
ON TOTALLY PERIODIC $\omega$-LIMIT SETS FOR MONOTONE MAPS ON REGULAR CURVES
}

\author{
AMIRA MCHAALIA
}

\begin{abstract}
An $\omega$-limit set of a continuous self-mapping of a compact metric space $X$ is said to be totally periodic if all of its points are periodic. In [3] Askri and Naghmouchi proved that if $f$ is one-to-one continuous self mapping of a regular curve, then every totally periodic $\omega$-limit set of $f$ is finite. This also holds whenever $f$ is a monotone map of a local dendrite by Abdelli in [1]. In this paper we generalize these results to monotone maps on regular curves. On the other hand, we give some remarks related to expansivity and totally periodic $\omega$-limit sets for any continuous map on compact metric space.
\end{abstract}

\section{Introduction}

The structure of $\omega$-limit sets plays an important role in studying dynamical system since it represents asymptotically the state of the orbit. A class of $\omega$-limit sets composed of periodic points called totally periodic $\omega$-limit is investigated. This notion was introduced by Marzougui and Naghmouchi in [11]. A compact metric space $X$ is said to have the $\omega$-FTP property if for each self continuous map $f$ of $X$, every totally periodic $\omega$-limit set of a point $x \in X$ is finite. We mention that totally periodic $\omega$-limit set appeared in the definition of $\omega$-chaos, introduced by $\mathrm{Li}$ in [10]. It also appeared as $\omega$-limit set of "spiral points" as proved in [2, Proposition 3.2] by Artigue. In zero-dimension, it was shown in [11] that, a zero dimensional compact metric space has the $\omega$-FTP property. In one-dimension, it was shown in ([1]) that any local dendrite has the $\omega$-FTP property when we restricted to monotone maps; that is any totally periodic $\omega$-limit set of a monotone map on a local dendrite is finite. The later result does not hold when we remove the restriction of monotonicity; it was shown in [11] that, there is a continuous map on a dendrite having an infinite totally periodic $\omega$-limit set. In [3]. it was shown that a regular curve has the $\omega$-FTP property when we restricted to one-to-one continuous maps. In this line, we generalize in the present paper the above results to monotone maps on regular curves.

Our main result is the following theorem.

Theorem 1.1. Let $X$ be a regular curve and $f: X \rightarrow X$ be a monotone map. Then any totally periodic $\omega$-limit set of $f$ is finite.

2000 Mathematics Subject Classification. 37B20, 37B45, 54H20.

Key words and phrases. Minimal set, regular curve, $\omega$-limit set, totally periodic, monotone map. 
In other words, a regular curve has the $\omega$-FTP property when we restricted to monotone maps.

Notice that Theorem 1.1 cannot be extended to other classes of systems such as rational curves; for instance, it was constructed in [13, Theorem D] an example of a homeomorphism of a rational curve (in fact a dendroid) for which some points have infinite $\omega$-limit sets consisting only of fixed points.

On the other hand, further results are obtained with regard to expansivity, we prove that any totally periodic $\omega$-limit set for expansive continuous map on a compact metric space $X$ is finite (see Proposition 5.2).

\section{Preliminaries}

Let $X$ be a compact metric space with metric $d$ and let $f: X \rightarrow X$ be a continuous map. Let $\mathbb{Z}, \mathbb{Z}_{+}$and $\mathbb{N}$ be the sets of integers, non-negative integers and positive integers, respectively. For $n \in \mathbb{Z}_{+}$denote by $f^{n}$ the $n$-th iterate of $f$; that is, $f^{0}=\mathrm{id}_{X}$ : identity of $\mathrm{X}$ and $f^{n}=f \circ f^{n-1}$ if $n \in \mathbb{N}$. A point $x \in X$ is called periodic of period $n \in \mathbb{N}$ if $f^{n}(x)=x$ and $f^{i}(x) \neq x$ for $1 \leq i \leq n-1$. If $n=1, x$ is a fixed point of $f$. For any $x \in X$, the subset $\operatorname{Orb}_{f}(x)=\left\{f^{n}(x): n \in \mathbb{Z}_{+}\right\}$is called the orbit of $x$ (under $f$ ). If $x$ is a periodic point, the orbit of $x$ is called periodic orbit. We denote by $P(f)$ and $\operatorname{Fix}(f)$ the set of periodic points and fixed points of $f$, respectively. A subset $A \subset X$ is called $f$-invariant if $f(A) \subset A$. It is called strongly $f$-invariant if $f(A)=A$. We define the $\omega$-limit set of a point $\mathrm{x} \in X$ to be the set

$$
\begin{aligned}
\omega_{f}(x) & =\left\{y \in X: \exists n_{i} \in \mathbb{N}, n_{i} \rightarrow+\infty, \lim _{i \rightarrow+\infty} d\left(f^{n_{i}}(x), y\right)=0\right\} \\
& =\bigcap_{n \in \mathbb{N}} \overline{\left\{f^{k}(x): k \geq n\right\}} .
\end{aligned}
$$

It is non-empty, closed and strongly $f$-invariant set (cf. [4, Chapter IV, Lemma 2]).

For any subset $A$ of $X$, we denote by $\bar{A}, \operatorname{Bd}(A)$ and $\operatorname{int}(A)$ the closure, boundary and interior of $A$, respectively. The symbol card $(A)$ stands for the cardinality of $A$ and $\operatorname{diam}(A)=\sup _{x, y \in A} d(x, y)$ means the diameter of $A$.

We denote by $B(x, \varepsilon)$ the open ball centered at $x$ of radius $\varepsilon$.

Definition 2.1 ([8], p. 131). Let $X, Y$ be two topological spaces. A continuous map $f: X \longrightarrow Y$ is said to be monotone if for any connected subset $C$ of $Y, f^{-1}(C)$ is connected.

When $f$ is closed and onto, Definition 2.1 is equivalent to that the preimage of any point by $f$ is connected (cf. [8], p. 131). Notice that $f^{n}$ is monotone for every $n \in \mathbb{N}$ when $f$ itself is monotone.

In the definitions below, we use the terminology from Nadler [12].

- A continuum is a compact connected metric space.

- An arc I (resp. a circle) is any space homeomorphic to the compact interval $[0,1]$ (resp. to the unit circle $\mathbb{S}^{1}=\{z \in \mathbb{C}:|z|=1\}$ ). For an arc 
$\mathrm{I}=[a, b]$, denote by $\gamma(I)=\{a, b\}$ the set of its endpoints, $[a, b)=\mathrm{I} \backslash\{b\}$ and $(a, b)=\mathrm{I} \backslash \gamma(I)=\mathrm{I} \backslash\{a, b\}$.

- A space is called degenerate if it is a single point, otherwise it is called non-degenerate.

- A dendrite is a locally connected continuum which contains no circle. - A local dendrite is a continuum such that every point of which has a dendrite neighborhood. A local dendrite is then a locally connected continuum containing only a finite number of circles (cf. [8], Theorem 4, p. 303).

- A regular curve is a continuum every point of which $x$ and each open neighborhood $V$ of $x$ in $X$, there exists an open neighborhood $U$ of $x$ in $X$ such that $U \subset V$ and the boundary set $\operatorname{Bd}(U)$ of $U$ is finite. Each regular curve is locally arcwise connected. Every sub-continuum of a regular curve is a regular curve (cf. [8] and [12]). Notice that every local dendrite is a regular curve (cf. [8], p. 303). There exists a regular curve which does not a local dendrites for example the Sierpińsky triangle.

- A hereditarily locally connected is a continuum such that every sub-continuum of it is locally connected.

- A Peano continuum is a locally connected continuum. In particular, each regular curve is a Peano continuum.

- A continuum $X$ is said to be a dendroid provided that $X$ is an arcwise connected hereditarily unicoherent space; that is if $X=A \cup B$ and $A, B$ are subcontinua of $X$, then $A \cap B$ is connected.

Given a compact metric space $X$ with metric $d$, we denote by $2^{X}$ (resp. $C(X)$ ) the set of all non-empty compact subsets (resp. continua) of $X$. We endow $2^{X}$ with the Hausdorff metric $d_{H}$ defined as follows:

For $A, B \in 2^{X}, d_{H}(A, B)=\max \left(\sup _{a \in A} d(a, B), \sup _{b \in B} d(b, A)\right)$, where $d(x, M)=\inf _{y \in M} d(x, y)$ for any $x \in X$ and $M \in 2^{X}$. For $A=\{a\}$, we write simply, $d_{H}(A, B)=d_{H}(a, B)$. It is well known that $\left(2^{X}, d_{H}\right)$ is a compact metric space (see [12], for more details). For any $B \in C(X)$, we denote by $\operatorname{mesh}(B)=\sup \{\operatorname{diam}(C): C$ is a connected component of $B\}$.

\section{Some results}

Lemma 3.1. Let $X$ be a compact metric space and $f$ be a continuous self-mapping of $X$. Then for all $n \in \mathbb{N}, P\left(f^{n}\right)=P(f)$.

Lemma 3.2. Let $X$ be a metric compact space and $f$ a continuous self mapping of $X$. Then for all $x \in X, \omega_{f}\left(f^{i}(x)\right)=\omega_{f}(x)$, for all $i \in \mathbb{N}$.

Lemma 3.3. ([4], p. 70) Let $X$ be a metric compact space and $f$ a continuous self mapping of $X$. Then for all $N \in \mathbb{N}$ and $x \in X$, $\omega_{f}(x)=\cup_{i=0}^{N-1} \omega_{f^{N}(x)}\left(f^{i}(x)\right)$.

Lemma 3.4. ([11, Theorem 1.1]) Let $X$ be a compact metric space, $f$ be a continuous self-mapping of $X$ and $x \in X$. If $\omega_{f}(x)$ is totally periodic, then $\omega_{f}(x)$ has finitely many connected components that form a periodic cycle. 
Recall that a $p$-tuple $\left(A_{0}, \ldots, A_{p-1}\right)$ of subsets of $X$ is called a periodic cycle if $f\left(A_{0}\right)=A_{1}, f\left(A_{1}\right)=A_{2}, \ldots, f\left(A_{p-1}\right)=A_{0}$.

Proposition 3.5. ([9, Theorem 1.6]) Let $\left(C_{n}\right)_{n \in \mathbb{N}}$ be a sequence of disjoint sub-continua of a regular curve $X$. Then $\lim _{n \rightarrow+\infty} \operatorname{diam}\left(C_{n}\right)=0$.

Lemma 3.6. ([8, Theorem 4, p. 257]) Let $X$ be a Peano continuum and $\varepsilon>0$ be a given number. Then there exists $\eta>0$ such that for every pair of points $(x, y)$ such that $d(x, y)<\eta$, there exists an arc I joining $x$ and $y$ with $\operatorname{diam}(I)<\varepsilon$.

Lemma 3.7. ([5, Lemma 4.2]) If $X$ is hereditarily locally connected, then for any sequence $\left(A_{n}\right)_{n \geq 0}$ in $C(X)$ that converges to $A \in C(X)$ (with respect to Hausdorff metric), we have $\lim _{n \rightarrow+\infty} \operatorname{mesh}\left(A_{n} \backslash A\right)=0$.

Lemma 3.8. Let $X$ be a regular continuum. Then for each sub-continuum $A$ of $X$, there is a decreasing sequence $\left(U_{n}\right)_{n \in \mathbb{N}}$ of open connected neighborhoods of $A$ with finite boundary such that $\lim _{n \rightarrow+\infty} \operatorname{mesh}\left(\mathrm{U}_{n} \backslash \mathrm{A}\right)=0$.

Proof. It is clear that

$$
A=\bigcap_{n \in \mathbb{N}} B\left(A, \frac{1}{n}\right), \text { where } B\left(A, \frac{1}{n}\right)=\left\{x \in X: d(x, A)<\frac{1}{n}\right\} .
$$

Since $A$ is compact and $X$ is locally connected, there exists, for each $n \in \mathbb{N}$, an open connected neighborhood $V_{n}$ of $A$ in $B\left(A, \frac{1}{n}\right)$ with finite boundary such that $A=\bigcap_{n \in \mathbb{N}} V_{n}$. For each $n \in \mathbb{N}$, set $W_{n}=\bigcap_{i=1}^{n} V_{i}$. Then $\left(W_{n}\right)_{n \in \mathbb{N}}$ is a decreasing sequence of open connected neighborhoods of $A$ and $A=\bigcap_{n \in \mathbb{N}} W_{n}$. Moreover $\operatorname{Bd}\left(W_{n}\right)$ is finite since each $\operatorname{Bd}\left(V_{i}\right)$ is finite, $1 \leq i \leq n$. Now, let $U_{n \in \mathbb{N}}$ be the connected component of $W_{n}$ containing $A$. Then the $\left(U_{n}\right)_{n \in \mathbb{N}}$ is a sequence of decreasing open connected neighborhoods of $A$ with $\operatorname{Bd}\left(U_{n}\right)$ finite and we have $A=\bigcap_{n \in \mathbb{N}} \bar{U}_{n}$. Since $X$ is locally connected, $U_{n}$ is open and connected. Moreover the sequence of continua $\left(\overline{U_{n}}\right)_{n \in \mathbb{N}}$ converges to $A$ in $C(X)$ (with respect to Hausdorff metric). By Lemma 3.7, we have that $\lim _{n \rightarrow+\infty} \operatorname{mesh}\left(\overline{U_{n}} \backslash A\right)=0$ and therefore $\lim _{n \rightarrow+\infty} \operatorname{mesh}\left(U_{n} \backslash A\right)=0$.

Lemma 3.9. Let $F$ be a non degenerate closed subset of $X$. Then

(1) $\eta(F):=\inf _{a \in F} d_{H}(a, F)>0$

(2) For any $a \in F$, there is $c \in F$ such that $d(a, c) \geq \eta(F)$.

Proof. (1) Since d $\mathrm{a}, \mathrm{F})=0$, for $a \in F$, so $d_{H}(a, F)=\sup _{b \in F} \mathrm{~d}(\mathrm{a}, \mathrm{b})$. There exists $a_{0} \in F$ and $b_{0} \in F$ such that $\inf _{a \in F} \mathrm{~d}_{H}(a, F)=d_{H}\left(a_{0}, F\right)=d\left(a_{0}, b_{0}\right)$. If $a_{0}=b_{0}$, so $d\left(a_{0}, b\right)=0, \forall b \in F$. This implies that $F=\left\{a_{0}\right\}$, a 
contradiction since $F$ is non degenerate. We conclude that $\eta(F)=\inf _{a \in F} d_{H}(a, F)>0$.

(2) For any $a \in F, d_{H}(a, F) \geq \eta(F)$ and so there is $c \in F$ such that $\mathrm{d}(a, c)=\mathrm{d}_{H}(a, F) \geq \eta(F)$.

\section{Proof of Theorem $\mathbf{1 . 1}$}

Suppose the theorem does not hold. Then we could find $x \in X$ such that $\omega_{f}(x)$ totally periodic (that is $\omega_{f}(x) \subset P(f)$ ) and infinite.

Lemma 4.1. Let $x \in X$ such that $\omega_{f}(x)$ is totally periodic. Then, there are $N \in \mathbb{N}$ and $0 \leq i \leq N-1$ such that:

(1) $\operatorname{int}_{\omega_{f^{N}}\left(f^{i}(x)\right)}\left(\operatorname{Fix}\left(f^{N}\right) \cap \omega_{f^{N}}\left(f^{i}(x)\right)\right) \neq \emptyset$.

(2) $\omega_{f^{N}}\left(f^{i}(x)\right)$ is connected.

Proof. (1) As $\omega_{f}(x)$ is totally periodic, so $\omega_{f}(x)=\cup_{n \in \mathbb{N}} \operatorname{Fix}\left(f^{n}\right) \cap \omega_{f}(x)$. By the Baire property, there is $N \in \mathbb{N}$ such that

$$
V:=\operatorname{int}_{\omega_{f(x)}}\left(\operatorname{Fix}\left(f^{N}\right) \cap \omega_{f}(x)\right) \neq \emptyset .
$$

As

$$
\omega_{f}(x)=\bigcup_{i=0}^{N-1} \omega_{f^{N}}\left(f^{i}(x)\right)(\text { Lemma 3.3), }
$$

so there exists $0 \leq i \leq N-1$ such that $V \cap \omega_{f^{N}}\left(f^{i}(x)\right) \neq \emptyset$.

As $V \cap \omega_{f^{N}}\left(f^{i}(x)\right) \subset \operatorname{Fix}\left(f^{N}\right) \cap \omega_{f^{N}}\left(f^{i}(x)\right)$, thus

$$
\operatorname{int}_{\omega_{f^{N}}\left(f^{i}(x)\right)}\left(\operatorname{Fix}\left(f^{N}\right) \cap \omega_{f^{N}}\left(f^{i}(x)\right)\right) \neq \emptyset .
$$

(2) Set $g=f^{N}$ and $y=f^{i}(x)$. From (1), $\operatorname{int}_{\omega_{g}(y)}\left(\operatorname{Fix}(g) \cap \omega_{g}(y)\right) \neq \emptyset$. We have $\omega_{g}(y) \subset \omega_{f}(y)=\omega_{f}(x)($ Lemma 3.2). As $P(f)=P(g)$ (Lemma 3.1), so $\omega_{g}(y) \subset P(g)$. Following Lemma $3.4, \omega_{g}(y)=\bigcup_{i=0}^{m-1} C_{i}$, for some $m \in \mathbb{N}$, where the $\left(C_{i}\right)_{0 \leq i \leq m-1}$ are the connected components of $\omega_{g}(y)$ with $g\left(C_{i}\right)=C_{i+1}$ for every $i \in\{0, \ldots, m-1\}$. As $\omega_{g}(y) \cap \operatorname{Fix}(g) \neq \emptyset$, so there is $a \in \omega_{g}(y) \cap \operatorname{Fix}(g)$. Therefore $a \in C_{i}$ for every $i \in\{0, \ldots, m-1\}$, which implies that $\omega_{g}(y)=C_{0}$. In result $\omega_{g}(y)$ is connected.

Set $g=f^{N}$ and $y=f^{i}(x)$. From Lemma 4.1, we have the following properties.

$-\omega_{g}(y) \subset P(g)$.

$-\operatorname{int}_{\omega_{g}(y)}\left(\operatorname{Fix}(g) \cap \omega_{g}(y)\right) \neq \emptyset$.

- $\omega_{g}(y)$ is a regular curve (since $\omega_{g}(y)$ is a sub-continuum of $X$ ). 
It follows that we can find an open connected set $\mathrm{O} \subset \operatorname{Fix}(g) \cap \omega_{g}(y)$. For the sequel, we let

$$
S_{a}=\cup_{i \geq 0} g^{-i}(a) \text {, for any } a \in \bar{O} .
$$

Lemma 4.2. Let $U$ be an open neighborhood of $\omega_{g}(y)$ in $X$ with finite boundary. Then there exists an open connected subset $V$ of $\omega_{g}(y)$ such that $\bar{V} \subset O$ and $S_{a} \subset U$, for all $a \in \bar{V}$.

Proof. First, the sets $\left(S_{a}\right)_{a \in \bar{O}}$ are pairwise disjoint connected sets; for $a \neq b \in \bar{O}$, we have $S_{a} \cap S_{b}=\emptyset$; indeed otherwise, there is $c \in S_{a} \cap S_{b}$, so $g^{j}(c)=a$ and $g^{i}(c)=b$ for some $j>i \in \mathbb{N}$. As $a, b \in \operatorname{Fix}(g)$, $a=g^{j-i}\left(g^{i}(c)\right)=g^{j-i}(b)=b$, a contradiction. By monotonicity of $g, S_{a}$ is connected. Set $\operatorname{card}(\operatorname{Bd}(U))=k$. So there are at most $k$ points $a_{1}, a_{2}, \ldots, a_{k} \in \bar{O}$ such that $S_{a_{i}} \not \subset U$ for every $i \in\{1, \ldots, k\}$ : Otherwise, there are at least $k+1$ points $a_{1}, a_{2}, \ldots, a_{k+1} \in \bar{O}$ such that $S_{a_{i}} \not \subset U$ for all $1 \leq i \leq k+1$. As $a_{i} \in S_{a_{i}} \cap U$ and $S_{a_{i}}$ is connected, so $S_{a_{i}}$ meets $\operatorname{Bd}(U)$ for every $1 \leq i \leq k+1$. Hence $\operatorname{card}(\mathrm{Bd}(\mathrm{U})) \geq k+1$, a contradiction. As $O \backslash\left\{a_{1}, a_{2}, \ldots, a_{k}\right\}$ is open in $\omega_{g}(y)$ and non-empty (since $\omega_{g}(y)$ is infinite), so we can find a non-empty open connected subset $V$ of $\omega_{g}(y)$ such that $\bar{V} \subset O \backslash\left\{a_{1}, a_{2}, \ldots, a_{k}\right\}$. This implies that for all $a \in \bar{V}, S_{a} \subset U$. The proof is complete.

Step 1. For $F=\overline{\mathrm{O}}$, we denote by $\eta:=\eta(F)>0$ be given as in Lemma 3.9. By Lemma 3.8, there exists a sequence of open connected neighborhoods $\left(U_{n}\right)_{n \in \mathbb{N}}$ of $\omega_{g}(y)$ in $X$ with finite boundary such that $\operatorname{mesh}\left(U_{n} \backslash \omega_{g}(y)\right)<\frac{\eta}{2}$. We let $U:=U_{N}$ and set card $(\operatorname{Bd}(\mathrm{U}))=k$. From Lemma 4.2, there exists an open connected subset $V \subset O$ of $\omega_{g}(y)$ such that for all $a \in \bar{V}, S_{a} \subset U$. Let $a \in V$. As $\{a\} \varsubsetneqq \omega_{g}(y)$, there is an open connected neighborhood $W$ of $a$ in $X$ such that $\bar{W} \cap \omega_{g}(y) \subset \bar{V}$ and $\omega_{g}(y) \backslash \bar{W} \neq \emptyset$. We can find two increasing sequences of integers $\left(n_{i}\right)_{i \in \mathbb{N}},\left(t_{i}\right)_{i \in \mathbb{N}}$ with the following properties:

(1) $g^{n_{i}}(y) \in W$ with $\lim _{i \rightarrow+\infty} g^{n_{i}}(y)=a$,

(2) $g^{n_{i}+j}(y) \in W$ for each $j=0, \ldots, t_{i}$, with $t_{i} \geq k$ and $t_{i} \geq i, \forall i \in \mathbb{N}$,

(3) $g^{n_{i}+t_{i}+1}(y) \in X \backslash W$ and since $b$ is a fixed point of $g$,

$$
\lim _{i \rightarrow+\infty} g^{n_{i}+t_{i}-k}(y)=b=\lim _{i \rightarrow+\infty} g^{n_{i}+t_{i}+1}(y) \in \bar{W} \backslash W .
$$

Observe that $a \in W \cap \omega_{g}(y)$ and $b \in(\bar{W} \backslash W) \cap \omega_{g}(y)$. So $a \neq b$.

We let $\mathbb{N}_{k}=\cup_{i \in \mathbb{N}}\left\{n_{i}, \ldots, n_{i}+t_{i}-k\right\}$. Since $\lim _{i \rightarrow+\infty} t_{i}=+\infty, \mathbb{N}_{k}$ is infinite.

By definition, $g^{m}(y) \in W$, for each $m \in \mathbb{N}_{k}$ and $\lim _{m \rightarrow+\infty, m \in \mathbb{N}_{k}} \mathrm{~d}\left(g^{m}(y), \bar{W} \cap \omega_{g}(y)\right)=0$. For each $m \in \mathbb{N}_{k}$, there is $b_{m} \in \bar{W} \cap \omega_{g}(y)$ such that $d\left(g^{m}(y), \bar{W} \cap \omega_{g}(y)\right)=d\left(g^{m}(y), b_{m}\right)$. Since $\bar{W}$ is a sub-continuum of $X$, so it is a Peano continuum. By Lemma 3.6, one can find an arc $\mathrm{T}_{m}=\left[b_{m}, g^{m}(y)\right] \subset \bar{W}$ such that $\lim _{m \rightarrow+\infty, m \in \mathbb{N}_{k}} \operatorname{diam}\left(T_{m}\right)=0$. 
Since $\omega_{g}(y)$ is infinite and $\omega_{g}(y) \subset P(g)$, so $g^{m}(y) \notin \omega_{g}(y)$, for every $m \in \mathbb{N}_{k}$.

Let $\mathrm{I}_{m}, m \in \mathbb{N}_{k}$, be the connected component of $\mathrm{T}_{m} \backslash \omega_{g}(y)$ containing $g^{m}(y)$. Then one can write $\mathrm{I}_{m}=\left[g^{m}(y), a_{m}\right)$ with $\overline{\mathrm{I}}_{m} \cap \omega_{g}(y)=\left\{a_{m}\right\}$. We see that $a_{m} \in \bar{W} \cap \omega_{g}(y) \subset \overline{\mathrm{V}} \subset \overline{\mathrm{O}} \subset \operatorname{Fix}(g) \cap \omega_{g}(y)$, for any $m \in \mathbb{N}_{k}$.

Claim 1. Under the notations above, there exists $N \in \mathbb{N}$ such that for all $m \in \mathbb{N}_{k}, m \geq N$, the family $F_{m}=\left\{I_{m+j}: 0 \leq j \leq k, m+j \in \mathbb{N}_{k}\right\}$ is not pairwise disjoint.

Proof. Suppose that, there exists an increasing sequence of positive integers $\left(m_{i}\right)_{i \geq 1}$ such that the family $F_{m_{i}}$ is pairwise disjoint. By passing to a subsequence of $\left(m_{i}\right)_{i \geq 1}$, one can assume that $\left(\overline{\mathrm{I}_{m_{i}}}\right)_{i \geq 1}$ converges to $\mathrm{I} \in C(X)$ (in Hausdorff metric). As $\lim _{i \rightarrow+\infty} \operatorname{diam}\left(T_{m_{i}}\right)=0$, then $\lim _{i \rightarrow+\infty} \operatorname{diam}\left(\overline{\mathrm{I}_{m_{i}}}\right)=0=\operatorname{diam}(\mathrm{I})$, so I is degenerate i.e. $\mathrm{I}=\{c\}$. As $g^{m_{i}}(y) \in \mathrm{I}_{m_{i}} \subset W$ and $\lim _{i \rightarrow+\infty} d_{H}\left(c, \overline{\mathrm{I}_{m_{i}}}\right)=0$, so $\lim _{i \rightarrow+\infty} g^{m_{i}}(y)=c \in \bar{W} \cap \omega_{g}(y)$. Since $\bar{W} \cap \omega_{g}(y) \subset \bar{V} \subset \bar{O} \subset \operatorname{Fix}(g)$, so for all $0 \leq j \leq k, \lim _{i \rightarrow+\infty} g^{m_{i}+j}(y)=g^{j}(c)=c$. In result, we have that $\lim _{i \rightarrow+\infty, m_{i}+j \in \mathbb{N}_{k}} \frac{i \rightarrow+\infty}{\mathrm{I}_{m_{i}+j}}=\{c\}$ (in Hausdorff metric). Now apply Lemma 3.9 for $c \in \bar{O}$ : there is $c^{\prime} \in \bar{O}$ such that $d\left(c, c^{\prime}\right) \geq \eta$. Then we can find two open neighborhoods $O_{c}$ and $O_{c^{\prime}}$ of $c$ and $c^{\prime}$ in $U$, respectively such that $d\left(O_{c}, O_{c^{\prime}}\right)>\frac{\eta}{2}$. As $c^{\prime} \in \bar{O} \subset \omega_{g}(y) \cap \operatorname{Fix}(g)$, so there is $m \geq 1$ such that $\left\{g^{m}(y), g^{m+1}(y), \ldots, g^{m+k}(y)\right\} \subset O_{c^{\prime}}$. Let $m_{p}>m$ such that

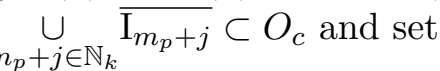

$$
C_{j}=g^{-\left(m_{p}-m\right)}\left(\overline{\mathrm{I}_{m_{p}+j}}\right), m_{p}+j \in \mathbb{N}_{k} .
$$

By monotonicity of $g, C_{j}$ is a sub-continuum of $X$. Then there is an arc $\mathrm{J}_{j}=\left[g^{m+j}(y), a_{m_{p+j}}\right] \subset C_{j}$. Each $J_{j}$ meets $O_{c}^{\prime}$ in at least $g^{m+j}(y)$ and meets $O_{c}$ in at least $a_{m_{p}+j} \in \operatorname{Fix}(g) \cap \omega_{g}(y)$. Let us show that $\mathrm{J}_{j} \backslash \omega_{g}(y)=\mathrm{J}_{j} \backslash\left\{a_{m_{p+j}}\right\}$ :

It is clear that $\mathrm{J}_{j} \backslash \omega_{g}(y) \subset \mathrm{J}_{j} \backslash\left\{a_{m_{p+j}}\right\}$. Conversely, if $z \in \mathrm{J}_{j} \cap \omega_{g}(y)$, then $g^{m_{p}-m}(z) \in \overline{\mathrm{I}}_{m_{p+j}} \cap \omega_{g}(y)=\left\{a_{m_{p+j}}\right\}$. As $a_{m_{p+j}} \in \operatorname{Fix}(g)$, then $g^{m_{p}-m}\left(a_{m_{p+j}}\right)=g^{m_{p}-m}(z)$. As $\omega_{g}(y) \subset P(g)$, then the restriction $g_{\mid \omega_{g}(y)}^{m_{p}-m}$ is a homeomorphism and therefore $z=a_{m_{p+j}}$. In result, $\mathrm{J}_{j} \backslash\left\{a_{m_{p+j}}\right\} \subset \mathrm{J}_{j} \backslash \omega_{g}(y)$ and hence the equality holds.

We conclude that $\mathrm{J}_{j} \backslash\left\{a_{m_{p+j}}\right\}$ is connected and hence $\operatorname{diam}\left(\mathrm{J}_{j}\right)>\frac{\eta}{2}$. Moreover we have that $J_{j} \not \subset U$ (since otherwise, $J_{j} \backslash\left\{a_{m_{p+j}}\right\} \subset U \backslash \omega_{g}(y)$ and so $\operatorname{diam}\left(J_{j} \backslash\left\{a_{m_{p+j}}\right\}\right) \leq \operatorname{mesh}\left(U \backslash \omega_{g}(y)\right)<\frac{\eta}{2}$. A contradiction). Therefore $C_{j} \cap \operatorname{Bd}(U) \neq \emptyset$, for each $m_{p}+j \in \mathbb{N}_{k}$.

Let us show that $C_{j} \cap C_{j^{\prime}} \cap \operatorname{Bd}(U)=\emptyset$ for all $j \neq j^{\prime}$. We have $\overline{\mathrm{I}_{m_{p}+j}} \cap \overline{\mathrm{I}_{m_{p}+j^{\prime}}} \subset\left\{a_{m_{p+j}}\right\}$ for $j \neq j^{\prime}$ (since $a_{m_{p+j}}=a_{m_{p+j^{\prime}}}$ ). Then $C_{j} \cap C_{j^{\prime}}=g^{-\left(m_{p}-m\right)}\left(\overline{\mathrm{I}}_{m_{p}+j} \cap \overline{\mathrm{I}}_{m_{p}+j^{\prime}}\right) \subset g^{-\left(m_{p}-m\right)}\left(a_{m_{p+j}}\right) \subset S_{a_{m_{p+j}}}$. As 
$a_{m_{p}+j} \in \bar{V}$, so by Lemma $4.2, S_{a_{m_{p+j}}} \subset U$. Hence $C_{j} \cap C_{j^{\prime}} \subset U$ and so $C_{j} \cap C_{j^{\prime}} \cap \operatorname{Bd}(U)=\emptyset$. We conclude that $\operatorname{Bd}(U)$ has at least $k+1$ points, which contradicts $\operatorname{Bd}(U)=k$. This completes the proof of Claim 1 .

Step 2. From Claim 1, there is some $m \in \mathbb{N}_{k}$ with $m+j_{1}(m), m+j_{2}(m) \in \mathbb{N}_{k}$ such that $\mathrm{I}_{m+j_{1}(m)} \cap \mathrm{I}_{m+j_{2}(m)} \neq \emptyset$. Hence $\mathrm{I}_{m+j_{1}(m)} \cup \mathrm{I}_{m+j_{2}(m)}$ is arcwise connected and so we can find an arc

$$
P_{m}=\left[g^{m+j_{1}(m)}(y), g^{m+j_{2}(m)}(y)\right] \subset \mathrm{I}_{m+j_{1}(m)} \cup \mathrm{I}_{m+j_{2}(m)} .
$$

We have that $P_{m} \cap \omega_{g}(y)=\emptyset$. Since $P_{m} \subset \mathrm{I}_{m+j_{1}(m)} \cup \mathrm{I}_{m+j_{2}(m)}$, we have $\lim _{m \rightarrow+\infty, m \in \mathbb{N}_{k}} \operatorname{diam}\left(P_{m}\right)=0$.

Claim 2. Let $B_{m}=g^{-j_{1}(m)}\left(P_{m}\right), m \in \mathbb{N}_{k}$. Then we have

$$
\lim _{m \rightarrow+\infty, m \in \mathbb{N}_{k}} \operatorname{diam}\left(B_{m}\right)=0 \text {. }
$$

Proof. Suppose that $\lim _{m \rightarrow+\infty, m \in \mathbb{N}_{k}} \operatorname{diam}\left(\mathrm{B}_{m}\right)>0$. Then there is $\delta>0$ and a sequence of positive integers $\left(m_{l}\right)_{l} \subset \mathbb{N}_{k}$ such that $\operatorname{diam}\left(\mathrm{B}_{m_{l}}\right)>\delta$, for all $l \in \mathbb{N}$. Recall $\lim _{m \rightarrow+\infty, m \in \mathbb{N}_{k}} \operatorname{diam}\left(\mathrm{I}_{m}\right)=0$, where $\mathrm{I}_{m}=\left[g^{m}(y), a_{m}\right)$ and $\lim _{l \rightarrow+\infty, m_{l} \in \mathbb{N}_{k}} \operatorname{diam}\left(\mathrm{P}_{m_{l}}\right)=0$. By passing to a subsequence if necessary, one can assume that $\left(\mathrm{P}_{m_{l}}\right)_{m_{l} \in \mathbb{N}_{k}}$ converges necessarily to a degenerate set, say $\{c\}$. Hence, $\lim _{l \rightarrow+\infty, m_{l} \in \mathbb{N}} g^{m_{l}+j_{1}\left(m_{l}\right)}(y)=c \in \omega_{g}(y)$. As $\mathrm{P}_{m_{l}} \cap \omega_{g}(y)=\emptyset$ for all $l \in \mathbb{N}$, so $c \notin \mathrm{P}_{m_{l}}$. Then we can construct a subsequence $\left(\mathrm{P}_{m_{l_{q}}}\right)_{q \geq 0, m_{l_{q}} \in \mathbb{N}_{k}}$ which is pairwise disjoint: indeed, as $\left(\mathrm{P}_{m_{l}}\right)_{m_{l} \in \mathbb{N}_{k}}$ converges to $\{c\}$, then for each neighborhood $U_{0}$ of $c$, there is $n_{0} \in \mathbb{N}$ such that for all $l \geq n_{0}, \mathrm{P}_{m_{l}} \subset U_{0}$. Since $c \notin \mathrm{P}_{m_{l}}$, for each $m_{l} \in \mathbb{N}_{k}$, so we can find an open neighborhood $U_{1} \subset U_{0}$ of $c$ such that $\mathrm{P}_{m_{n_{0}}} \nsubseteq U_{1}$. Moreover, since $\left(\mathrm{P}_{m_{l}}\right)_{m_{l} \in \mathbb{N}_{k}}$ converges to $\{c\}$, so there is $n_{1} \in \mathbb{N}$ such that for all $l \geq n_{1}, \mathrm{P}_{m_{l}} \in U_{1}$, we obtain $\mathrm{P}_{m_{n_{0}}} \cap \mathrm{P}_{m_{n_{1}}}=\emptyset$. We continue in this fashion obtaining a subsequence $\left(\mathrm{P}_{m_{l_{q}}}\right)_{q \geq 0, m_{l_{q}} \in \mathbb{N}_{k}}$ which is pairwise disjoint. Since $0 \leq j_{1}\left(m_{l_{q}}\right) \leq k$, we can assume that $j_{1}\left(m_{l_{q}}\right)=j_{0}$, for all $q \in \mathbb{N}$. Then the $\mathrm{B}_{m_{l_{q}}}=g^{-j_{0}}\left(\mathrm{P}_{m_{l_{q}}}\right), q \in \mathbb{N}$, are connected and pairwise disjoint sets. Hence, by Proposition 3.5, $\lim _{q \rightarrow+\infty, m_{l_{q}} \in \mathbb{N}_{k}} \operatorname{diam}\left(\mathrm{B}_{m_{l_{q}}}\right)=0$. This contradicts the fact that $\operatorname{diam}\left(\mathrm{B}_{m_{l}}\right)>\delta$, for all $l \in \mathbb{N}, m_{l} \in \mathbb{N}_{k}$. This completes the proof.

End of the proof of Theorem 1.1. Recall that $\omega_{g}(y) \subset P(g)$ and $\omega_{g}(y) \cap \operatorname{Fix}(g) \neq \emptyset$ is infinite. From the notation given in Step 1, we have that $\lim _{i \rightarrow+\infty} g^{n_{i}}(y)=a$ and $\lim _{i \rightarrow+\infty} g^{n_{i}+t_{i}-k}(y)=b$ with $a \neq b \in \omega_{g}(y) \cap \operatorname{Fix}(g)$. From Claim 2, $\mathrm{B}_{m}=g^{-j_{1}(m)}\left(P_{m}\right)$ is connected for each $m \in \mathbb{N}_{k}$ and contains the points $g^{m}(y)$ and $g^{m+j_{2}(m)-j_{1}(m)}(y)$. Moreover, for each $m \in \mathbb{N}_{k}$, we have $B_{m} \cap \omega_{g}(y)=\emptyset$; otherwise, there is $m \in \mathbb{N}_{k}$ such that $z \in B_{m} \cap \omega_{g}(y)$ and so $g^{j_{1}(m)}(z) \in P_{m} \cap \omega_{g}(y)$, this leads to a contradiction 
with $P_{m} \cap \omega_{g}(y)=\emptyset$. By Claim 2, $\lim _{m \rightarrow+\infty, m \in \mathbb{N}_{k}} \operatorname{diam}\left(\mathrm{B}_{m}\right)=0$. So let $W_{a}$, $W_{b}$ be two open neighborhoods of $a$ and $b$, respectively such that $d\left(W_{a}, W_{b}\right)>\frac{d(a, b)}{2}$. By Lemma 3.8, there exists a connected neighborhood $U^{\prime}:=U_{N^{\prime}}$ of $\omega_{g}(y)$ such that $\operatorname{mesh}\left(U^{\prime} \backslash \omega_{g}(y)\right)<\frac{d(a, b)}{2}$. Since $\lim _{m \rightarrow+\infty} d\left(g^{m}(y), \omega_{g}(y)\right)=0$, so there is $m_{0} \in \mathbb{N}$ such that for all $m \geq m_{0}$, $m \in \mathbb{N}_{k}, B_{m} \subset U^{\prime}$. Thus there exists $q \in \mathbb{N}$ such that for $n_{q} \geq m_{0}$, $g^{n_{q}}(y) \in W_{a}$ and $\left\{g^{n_{q}+t_{q}-k}(y), \ldots, g^{n_{q}+t_{q}}(y)\right\} \subset W_{b}$.

For each $m \in \mathbb{N}_{k}$, denote by

$-s(m)=m+j_{2}(m)-j_{1}(m)$, where $m+j_{1}(m), m+j_{2}(m) \in \mathbb{N}_{k}$,

$-p_{n_{q}}=\inf \left\{i \in \mathbb{N}: s^{i}\left(n_{q}\right) \in\left\{n_{q}+t_{q}-k+1, \ldots, n_{q}+t_{q}\right\}\right\}$,

$-K_{q}=\mathrm{B}_{n_{q}} \cup \mathrm{B}_{s\left(n_{q}\right)} \cup \cdots \cup \mathrm{B}_{s^{p_{n_{q}}-1}\left(n_{q}\right)}$.

It is easy to see that $K_{q}$ is connected. Since for all $0 \leq i<p_{n_{q}}$, $s^{i}\left(n_{q}\right) \in \mathbb{N}_{k}$, so $\mathrm{B}_{s^{i}\left(n_{q}\right)} \cap \omega_{g}(y)=\emptyset$ and then $K_{q} \cap \omega_{g}(y)=\emptyset$. Moreover as $K_{q}$ meets $W_{a}$ in at least $g^{n_{q}}(y)$ and meets $W_{b}$ in at least $g^{s_{n_{n_{q}}}}(y)$, thus $\operatorname{diam}\left(K_{q}\right)>\frac{d(a, b)}{2}$. Since for all $n_{q} \geq m_{0}, B_{n_{q}} \subset U^{\prime}, K_{q} \subset U^{\prime}$ and $K_{q} \cap \omega_{g}(y)=\emptyset$, thus $K_{q} \subset U^{\prime} \backslash \omega_{g}(y)$. Therefore $\operatorname{diam}\left(K_{q}\right) \leq \operatorname{mesh}\left(U^{\prime} \backslash \omega_{g}(y)\right)<\frac{d(a, b)}{2}$. A contradiction.

\section{Expansivity and totally periodic $\omega$-limit sets}

In this section, we consider the notion of expansivity of continuous maps on compact metric space.

Definition 5.1. [14] A continuous map $f: X \longrightarrow X$ on a compact metric space $X$ is said to be expansive if there is real constant $c>0$ such that for any $x, y \in X$ and $x \neq y$, there is an integer $n \in \mathbb{Z}_{+}$such that $d\left(f^{n}(x), f^{n}(y)\right)>c$.

It is well known that the Cantor set and the torus $\mathbb{T}^{2}$ admit expansive homeomorphisms [15]. Kawamura showed [7] that a Peano continuum which contains a free arc admits no expansive homeomorphisms. In particular, there is no expansive homeomorphism on an arc and a circle. In [6], Kato showed that no dendroid admits an expansive homeomorphism. With respect to expansivity, we show the following.

Proposition 5.2. If $f: X \longrightarrow X$ is an expansive continuous map on a compact metric pace $X$, then any totally periodic $\omega$-limit set is finite.

Lemma 5.3. ([4, Lemma 4]) Let $(X, f)$ be a dynamical system and let $x \in X$ such that $\omega_{f}(x)$ is infinite. If $z$ is an isolated point in $\omega_{f}(x)$, then it is not periodic.

Proof of Proposition 5.2. Let $x \in X$ such that $\omega_{f}(x) \subset P(f)$ and assume that $\omega_{f}(x)$ is infinite. We have that $\omega_{f}(x)=\cup_{n \in \mathbb{N}} \operatorname{Fix}\left(f^{n}\right) \cap \omega_{f}(x)$. Then we claim that there exists $N \in \mathbb{N}$ such that $\operatorname{Fix}\left(f^{N}\right) \cap \omega_{f}(x)$ is infinite. 
Otherwise, $\omega_{f}(x)$ would be compact countable, so it contains an isolated point $a$ and therefore by Lemma 5.3, $a \notin P(f)$. A contradiction.

Now let $\left(x_{n}\right)_{n \in \mathbb{N}} \subset \operatorname{Fix}\left(f^{N}\right) \cap \omega_{f}(x)$ be an infinite sequence which converges to some point $y$. From expansivity of $f$, there is a constant $c>0$ such that for any $n \in \mathbb{N}$, there is an integer $k_{n}$ such that $d\left(f^{k_{n}}\left(x_{n}\right), f^{k_{n}}\left(x_{n+1}\right)\right)>c$. One can write $k_{n}=s_{n} N+r_{n}$ with $0 \leq r_{n}<N$. We may assume, by passing to a subsequence if necessary, that $r_{n}=r$, for every $n$. It follows that $d\left(f^{r}\left(x_{n}\right), f^{r}\left(x_{n+1}\right)\right)>c$, for every $n$ and so letting $n \rightarrow+\infty$, we obtain that $d\left(f^{r}(y), f^{r}(y)\right)=0 \geq c$. A contradiction.

In [2], Artigue introduced the notion of turning point for a continuous map $f: X \rightarrow X$ on a metric space $(X, d)$ as follows: we say that $x \in X$ is a spiral point of $f$ if there is $m \in \mathbb{N}$ such that $\lim _{n \rightarrow+\infty} d\left(f^{n}(x), f^{n+m}(x)\right)=0$. From [2, Proposition 3.2] and Proposition 5.2, we obtain:

Corollary 5.4. If $f: X \rightarrow X$ is an expansive continuous map on a metric space $(X, d)$ and $x \in X$ is a spiral point of $f$, then $\omega_{f}(x)$ is finite.

Acknowledgements. This work was supported by the research unit: "Dynamical systems and their applications", (UR17ES21), Ministry of Higher Education and Scientific Research, Faculty of Science of Bizerte, Bizerte, Tunisia.

\section{REFERENCES}

[1] H. Abdelli, $\omega$-limit sets for monotone local dendrite maps, Chaos Solitons Fractals 71 (2015), 66-72.

[2] A. Artigue, Minimal expansive systems and spiral points, Topology Appl., 194 (2015), 166-170.

[3] G. Askri and I. Naghmouchi, On totally periodic $\omega$-limit sets in regular continua, Chaos Solitons Fractals 75 (2015), 91-95.

[4] L.S. Block, W.A. Coppel, Dynamics in One Dimension, Lecture Notes in Math, 1513. Springer-Verlag, 1992.

[5] A. Daghar (2021): On regular curve homeomorphisms without periodic points, Journal of Difference Equations and Applications, DOI: 10.1080/10236198.2021.1912030.

[6] H. Kato, The nonexistence of expansive homeomorphisms on dendroids, Fund. Math. 136 (1990), 37-43.

[7] K. Kawamura, A direct proof that each Peano continuum with a free arc admits no expansive homeomorphisms, Tsukuba J. Math., 12 (1988), 521-524.

[8] K. Kuratowski, Topology, vol.2, Academic Press, New-York, 1968.

[9] A. Lelek, On the topology of curves. II. Fund. Math. 70, (1971), 131-138.

[10] S. Li, $\omega$-chaos and topological entropy, Trans. Amer. Math. Soc., 339 (1993), 243-249.

[11] H. Marzougui and I. Naghmouchi, On totally periodic $\omega$-limit sets, Houston J. Mathematics 43 (2017), 1291-1303.

[12] S.B. Nadler, Continuum Theory: An Introduction, (Monographs and Textbooks in Pure and Applied Mathematics, 158). Marcel Dekker, Inc., New York, 1992.

[13] I. Naghmouchi, Dynamics of monotone graph, dendrite and dendroid maps, Internat. J. Bifur. Chaos Appl. Sci. Engrg., 21 (2011), 3205-3215.

[14] W. Utz, Unstable homeomorphisms, Proc. Amer. Math. Soc., 1 (1950), 769-774. 
[15] R.F. William, A note on unstable homeomorphisms, Proc. Amer. Math. Soc., 6 (1955), 308-309.

Amira Mchaalia, University of Carthage, Faculty of Science of Bizerte, (UR17ES21), "Dynamical Systems and their applications", Jarzouna, 7021, BizerTe, Tunisia.

Email address: emira.mchaalia@gmail.com 